\title{
Pulmonary embolism in transit: direct visualization of a PE passing through the heart using bedside ultrasonography
}

\author{
Daniel Joseph, MD*; Evelyn Lee, MD*; Ramin Tabatabai, MD*; Mikaela Chilstrom, MD*
}

A 59-year-old man with a history of atrial fibrillation presented to the emergency department with several days of progressive dyspnea and three episodes of syncope. On physical examination, blood pressure was 154/124 mm Hg, pulse 149 beats/min, respiratory rate 28 breaths/min, and oxygen saturation $83 \%$ on room air. He was diaphoretic (but speaking fluently), had clear lungs, a rapid, irregular heartbeat without murmurs, and bilateral lower extremity edema. An electrocardiogram confirmed atrial fibrillation with rapid ventricular response, and a clinical ultrasound demonstrated no pericardial effusion, a moderately decreased left ventricular ejection fraction, no right ventricular (RV) strain, and a hyperechoic, mobile,

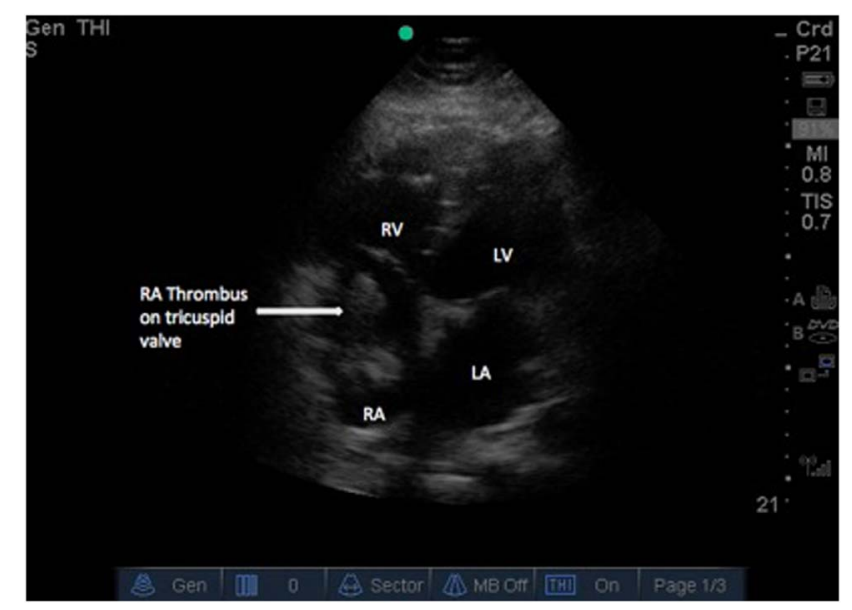

Figure 1. Apical four chamber view of the heart demonstrating a large thrombus in the right atrium extending across the tricuspid valve. $\mathrm{RA}=$ right atrium, $\mathrm{RV}=$ right ventricle, $\mathrm{LA}=$ left atrium, $\mathrm{LV}=$ left ventricle. serpiginous mass oscillating between the right atrium and right ventricle (Figures 1 and 2). Chest computed tomography (Figure 3) was performed to confirm the diagnosis and evaluate the extent of disease.

Pulmonary embolism (PE) in transit refers to the direct visualization of distal thrombi passing through the right side of the heart. Cases with this echocardiogram finding are associated with significantly worse mortality. ${ }^{1}$ Recommendations are limited, but favor thrombolysis or embolectomy rather than anticoagulation alone. ${ }^{2}$ Although typical echocardiogram findings suggestive of $\mathrm{PE}$ include $\mathrm{RV}$ strain and McConnell's sign (RV free wall hypokinesis with preserved apical contractility), ${ }^{3}$ this patient had neither of these findings, but the presence of a right-sided thrombus confirmed the likely diagnosis of $\mathrm{PE}$ and

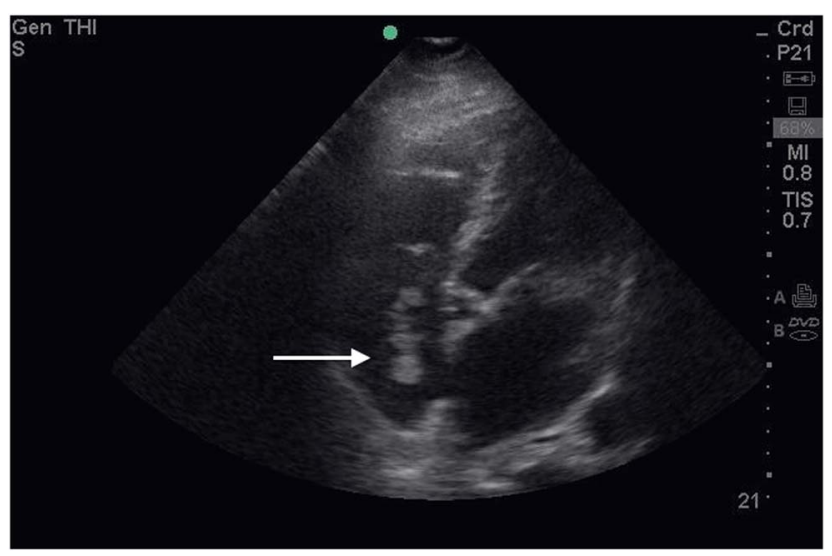

Figure 2. Apical five chamber view of the heart demonstrating thrombus in the right atrium (white arrow).

From the *Department of Emergency Medicine, LA County USC Medical Center, Los Angeles, CA, USA.

Correspondence to: Dr. Daniel Joseph, Department of Emergency Medicine, Keck School of Medicine of the University of Southern California, 1200 N. State St., Room 1060H, Los Angeles, CA 90033-1029; Email: daniel.adrian.joseph@ gmail.com 


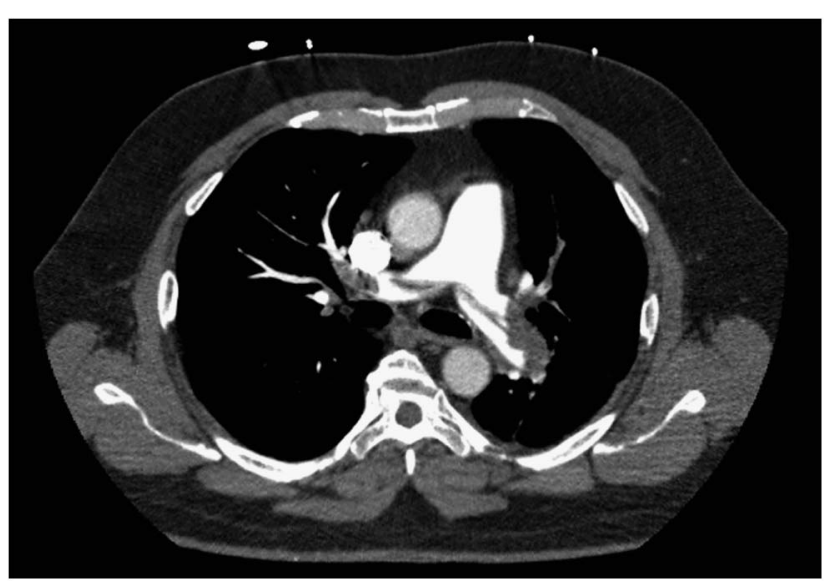

Figure 3. Axial CT of the chest with contrast demonstrating pulmonary emboli in the right and left main pulmonary arteries.

allowed for prompt management. He received anticoagulation with heparin as well as fibrinolytics. His respiratory status improved throughout his hospitalization, and he was discharged on warfarin with cardiology and pulmonary follow-up.

Competing interests: None declared.

Keywords: Pulmonary embolism, pulmonary embolism in transit, echocardiography, point-of-care systems

\section{REFERENCES}

1. Torbicki A, Galié N, Covezzoli A, et al. ICOPER Study Group. Right heart thrombi in pulmonary embolism: results from the International Cooperative Pulmonary Embolism Registry. 7 Am Coll Cardiol 2003; 41(12):2245-51.

2. Fischer JI, Huis in 't Veld MA, Orland M, et al. Diagnosis of near-fatal pulmonary embolus-in-transit with focused echocardiography. 7 Emerg Med 2013;45(2):232-5.

3. McConnell MV, Solomon SD, Rayan ME, et al. Regional right ventricular dysfunction detected by echocardiography in acute pulmonary embolism. Am 7 Cardiol 1996;78(4): 469-73. 